\title{
Ecology/Ecologia \\ Borboletas frugívoras de fragmentos de Mata Atlântica no noroeste do Rio Grande do Sul, Brasil (Lepidoptera: Nymphalidae)
}

\author{
Thamyrys Pinho da Silva ${ }^{\bowtie}$, Willian Falcão Pias \& Ricardo Giovenardi
}

Universidade Regional Integrada do Alto Uruguai e das Missões.

\author{
EntomoBrasilis 11 (2): 95-102 (2018)
}

\begin{abstract}
Resumo: O presente estudo teve como objetivo principal fornecer uma lista e avaliar a diversidade das espécies de borboletas frugívoras em dois fragmentos de Floresta Estacional Decidual no noroeste do Rio Grande do Sul. As amostragens foram mensais entre junho de 2014 a março de 2015. Para a captura das borboletas foram utilizadas armadilhas com isca atrativa à base de banana fermentada em caldo de cana. Após um esforço amostral de 4800 horas, foram amostrados 760 indivíduos distribuídos em 49 espécies de borboletas. Satyrinae foi a subfamília com maior riqueza e abundância de espécies. Hypothyris euclea laphria (Doubleday) representou um novo registro para o estado. A abundância de borboletas apresentou-se de forma heterogênea em ambos os fragmentos no decorrer dos meses amostrados. Apesar de ser um inventariamento de curto prazo, a riqueza de borboletas foi bastante representativa no noroeste do Rio Grande do Sul. Recomenda-se intensificar estudos semelhantes, para prover informações no intuito de conservação dos hábitats.
\end{abstract}

Palavras-chave: Borboletas; Conservação; Inventário; Mata Atlântica; Riqueza de espécies.

\section{Butterflies from Atlantic Forest fragments in northwest of Rio Grande do Sul, Brasil (Lepidoptera: Nymphalidae)}

Abstract. This study aimed to provide a list and evaluate the diversity of species of fruit-feeding butterflies in two fragments of Decidual Seasonal Forest in the northwest of Rio Grande do Sul. Samples were collected monthly, between June 2014 and March 2015. Traps with attractive baits of fermented banana in sugar cane juice were used to capture the butterflies. After a 4800 hours sampling effort, 760 butterflies were sampled and distributed into 49 species. Satyrinae was the family with the greatest wealth and abundance of species. Hypothyris euclea laphria (Doubleday) represented a new registry for the state. The abundance of butterflies was heterogeneous in both fragments during the months sampled. Despite being a short-term inventory, butterfly richness was quite representative in the northwest of Rio Grande do Sul. It is recommended to intensify similar studies to provide information for the conservation of habitats.

Keywords: Butterflies; Conservation; Inventary; Mata Atlântica; Species richness.

Q onsiderado um dos mais importantes "hotspots" tropicais, o bioma Mata Atlântica ocupa aproximadamente $15 \%$ do território nacional (Ministério Do Meio Ambiente 2010), e hoje com a pressão antrópica, apresenta apenas $27 \%$ de sua cobertura original preservada (MinistéRIo do MeIo Ambiente 2017). Atualmente, os remanescentes de Floresta Atlântica ocorrem principalmente em pequenos fragmentos de forma isolada (Ribeiro et al. 2009), resultando a perda de quase todas das florestas originais e consequentemente, a perda da biodiversidade (Ministério do MeIo AmbIENTE 2017). Entretanto, é um ambiente altamente heterogêneo que oferece grande variedade de fitofisionomias e composições florestais abrigando 1-8\% de todas as espécies mundiais (Ribeiro et al. 2009).

Muitas vezes as consequências do desmatamento podem não ser percebidas em primeira instância, no entanto, processos ecológicos são gravemente atingidos com o fenômeno da fragmentação, o que pode comprometer o fornecimento de serviços ambientais por meio das espécies e suas interações (Ministério do Meio Ambiente 2010). Diante do exposto, a conservação desses fragmentos é de essencial importância para estabelecer corredores naturais de vegetação e a conexão das paisagens, visando à conservação da biodiversidade (BROwn \& FREITAS 2000).

O filo artrópode, maior filo animal compreendendo mais de 800 mil espécies vem sendo amplamente utilizado para avaliar a composição e diversidade de diferentes hábitats frente a modificações da biota onde estão inseridos (РЕDROTTI et al. 2011). Dentro deste grupo, os insetos representam quase 60\% de todos os animais do planeta (SiLva et. al. 2013), os quais desenvolvem papel chave nos ecossistemas terrestres, inclusive para o homem
Edited by:

William Costa Rodrigues

\section{Article History:}

Received: 27.ii.2018

Accepted: 19.iv.2018

\section{Corresponding author:}

Thamyrys Pinho da Silva

○thamygnr@hotmail.com

( No ORCID record
Funding agencies:

$\diamond$ Without funding declared 
que se beneficia de práticas como a dispersão de sementes e a polinização (FreITAS et al. 2011).

Os insetos são parte vital dos ciclos naturais devido ao fato que a maior parte do fluxo de energia contido nos níveis tróficos pode estar ligada a uma fase da vida destes organismos (BRANCHER \& RosA-Gomes 2012). Entre os insetos, as borboletas são de suma importância em estudos conservacionistas, principalmente em ambientes fragmentados ou antropizados, fornecendo dados sobre perturbações e processos ambientais (FreITAS et al. 2003).

A guilda de borboletas frugívoras é representada por quatro subfamílias pertencentes à família Nymphalidae: Satyrinae, Charaxinae, Biblidinae e Nymphalinae (WAHLBERg et al. 2009). A principal característica das borboletas frugívoras é a alimentação através das frutas fermentadas, sendo facilmente amostradas com a utilização de iscas atrativas (DEVRIES 1997).

Até a década de 90, a maioria dos trabalhos no Rio Grande do Sul foram realizados com metodologias não padronizadas (PEDRotTi et al. 2011) e na atualidade, em determinadas regiões, são inexistentes as informações sobre a fauna de borboletas. Os trabalhos efetivados incluem listagem de espécies revisando coleções científicas, não contemplando, em muitos momentos, informações importantes sobre o local, períodos de coleta e o esforço amostral utilizado (ISERHARD \& ROMANOWSKI 2004).

No noroeste do Rio Grande do Sul não há pesquisas envolvendo a fauna de borboletas frugívoras, acarretando em uma subamostragem do grupo, levando a desconsideração de informações da composição das espécies. A partir disto, o presente trabalho possui como objetivo fornecer uma lista e avaliar a diversidade das espécies de borboletas frugívoras em dois fragmentos de Floresta Estacional Decidual no noroeste do Rio Grande do Sul.

\section{MATERIAL E MÉTODOS}

Local de estudo. Localizado na região do Alto Uruguai, o município de Frederico Westphalen (27 $21^{\circ} \mathrm{S}$ e $53^{\circ} 23^{\prime} \mathrm{W}$ ) caracteriza-se por vegetação denominada Floresta Estacional Decidual com as estações anuais bem definidas e perda de cobertura foliar durante a estação de seca. O clima é subtemperado subúmido com a temperatura média anual de $18,8^{\circ} \mathrm{C}$ e a precipitação anual de $1.787 \mathrm{~mm}$ (MALuF 2000). O estudo foi realizado em dois fragmentos localizados no município de Frederico Westphalen, denominados Parque Municipal

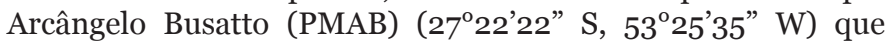
possui aproximadamente 35 ha, e Santuário de Schoenstatt (SS) ( $27^{\circ} 22$ ' $\left.37^{\prime \prime} \mathrm{S}, 53^{\circ} 24^{\prime} 41^{\prime \prime} \mathrm{W}\right)$ com aproximadamente 50 ha (Figura 1). Ambos os fragmentos apresentam perturbação ambiental devido a fatores antrópicos, pois são circundados por lavouras de diversos cultivos e são frequentados por pessoas nos finais de semana.

Amostragem. As amostragens foram mensais entre junho de 2014 a março de 2015. Foram utilizadas 12 armadilhas com iscas atrativas em cada fragmento. As armadilhas seguiram modelo adaptado de UEHARA-Prado et al. (2005). O material utilizado como isca atrativa foi um composto de caldo de cana e banana fermentado por $48 \mathrm{~h}$. As armadilhas foram instaladas nas trilhas dos fragmentos onde permaneceram dois dias ao mês e revisadas em 48 horas em cada ocasião amostral. O número de horas de amostragem foi calculado multiplicando-se o número de armadilhas pelo número de dias de amostragem e pelo período de $10 \mathrm{~h}$ (tempo ao longo do dia que supõe, que as borboletas estejam ativas e possam ser atraídas pela isca) (PEDRotTi et al. 2011). As borboletas não identificadas em campo foram coletadas e armazenadas individualmente em envelopes entomológicos para posterior montagem e identificação no Laboratório de Morfologia da Universidade Regional Integrada do Alto Uruguai e das Missões - Campus Frederico Westphalen. O material foi identificado por meio de bibliografia especializada (CANals 2000, 2003; Uehara-Prado et al. 2004; Penz et al. 2007). A nomenclatura utilizada seguiu LAMAS (2004) e WAHLBERG et al. (2009). As coletas foram executadas com amparo da licença expedida pelo ICMBio (Licença de coleta ICMBio/SISBIO 44276-2).

Análise dos dados. A listagem de espécies deste estudo foi comparada com outros inventários já realizados envolvendo a família Nymphalidae para o Rio Grande do Sul, com a finalidade de confirmar novos registros (BIEZANKo 1960a; BiEZANKo 196ob; Link \& Alvarez-Filho 1979; Link et al. 1980; Teston \& Corseuil 2002a; TESTON \& CORSEUIL 2002b; ISERHARD \& ROMANOWSKI 2004; Quadros et al. 2004; MARChiori \& Romanowski 2006; Dessuy \& Morais 2007; GiovenARdi et al. 2008; Lemes et al. 2008; Paz et al. 2008; SACKis \& Morais 2008; Teston \& Corseuil 2008a; Teston \& Corseuil 2008b; Teston \& Corseull 2008c; Bonfantti et al. 2009; ROMANOWSKI et al. 2009; ISERHARD et al. 2010; PEDROTTI et al. 2011; RITTER et al. 2011; Rosa et al. 2011; SANTOs et al. 2011; BELLAVER et al. 2012; Morais et al. 2012; Silva et al. 2013; MARCHIORI et al.

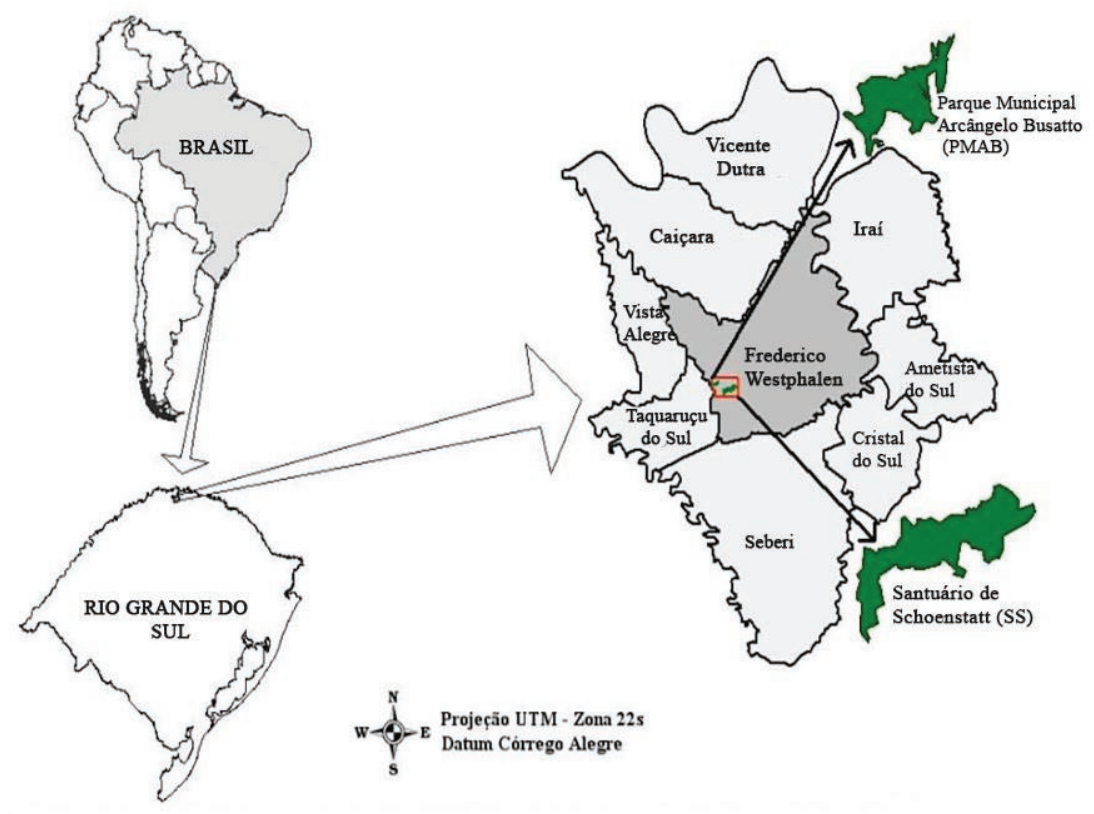

Figura 1. Localização das áreas de estudo. PMAB (Parque Municipal Archângelo Busatto), SS (Santuário de Schoenstatt). Fonte: adaptado do Laboratório de Geoprocessamento da URI - Campus de Frederico Westphalen. 
2014; Paz et al. 2014; Thiele et al. 2014; CARPorale et al. 2015; CARValho \& Morais 2015; CARVAlho et al. 2015; Lemes et al. 2015; Piovesan \& Morais 2015; Spaniol \& Morais 2015). Para a análise faunística, foram considerados os valores de riqueza de espécies (S) e abundância relativa $(\mathrm{N})$ para cada fragmento. Foram consideradas dominantes as espécies com frequência relativa maior que 0,10 (Dessuy \& Morais 2007). A suficiência amostral foi mensurada através de curva de acumulação de espécies com os respectivos intervalos de confiança a 95\% de probabilidade e dos estimadores analíticos de riqueza Jackknife 1, Jackknife 2 e Bootstrap. O critério para escolha do melhor estimador baseouse no fato dele se apresentar estável independentemente do tamanho da amostra, possuir estabilidade no desvio padrão e estabilizar-se com o mínimo possível de esforço amostral (SPANIOL \& MoraIs 2015). A abundância das borboletas entre os meses de amostragens para cada fragmento foi comparada com o teste do qui-quadrado $\left(\chi^{2}\right)$. Todas as análises foram realizadas com o software PAST 1.75 (Hammer et al. 2001).

\section{RESULTADOS E DISCUSSÃO}

Em 4.80o horas de amostragem foram registrados 760 indivíduos distribuídos em 49 espécies/subespécies de borboletas (Tabela 1). Destes, 454indivíduos pertencemasubfamília Satyrinae(59,74\%), 177 Biblidinae (23,29\%), 55 Morphinae (7,24\%), 49 Charaxinae (6,45\%), 17 Nymphalinae (2,24\%), cinco Ithomiinae (o,66\%), e três Liminitidinae (o,39\%) (Figura 2). Até o momento, essa foi a segunda maior riqueza de borboletas frugívoras registrada no Rio Grande do Sul, comparando com trabalhos que utilizaram metodologias semelhantes do presente estudo (PеDrotTi et al. 2011; Silva et al. 2013; Paz et al. 2014; SPANiol \& Morais 2015). A diferença entre os esforços amostrais não permite comparações mais detalhadas. Recentemente, no município de Santa Maria, em duas áreas com influência do Bioma Mata Atlântica, foram registradas 36 espécies de borboletas com este mesmo método de estudo (Spaniol \& Morais 2015).

As borboletas frugívoras representam 52,69\% do total de Nymphalidae quando comparadas com outros trabalhos desenvolvidos na mesma região (Giovenardi et al. 2008; BonfANTTI et al. 2009). Este resultado é semelhante ao que outros estudos relatam (40 a 55\%) quanto ao percentual de espécies de frugívoras encontradas em florestas tropicais (PINHEIRO \& ORTIZ 1992; Daily \& Ehrlich 1995; DeVries et al. 1999; DeVries \& WALLA 2001).

Satyrinae foi a subfamília com maior riqueza apresentando 18 espécies (36,73\%), posteriormente, 14 Biblidinae (28,57\%), quatro Charaxinae (8,16\%), quatro Morphinae $(8,16 \%)$, quatro Nymphalinae $(8,16 \%)$, quatro Ithomiinae $(8,16 \%)$ e um Liminitidinae (2,04\%) (Figura 2). Segundo (Brown \& FreITAS 2000), Satyrinae é a subfamília mais abundante em ambientes neotropicais, mesmo apresentando sensibilidade ao processo de fragmentação. A maior representatividade de riqueza de Satyrinae segue os padrões que já observados para o Rio Grande do Sul (PeDrotti et al. 2011; SANtos et al. 2011; Bellaver et al. 2012; SiLva et al. 2013).

Uma subespécie de Satyrinae que merece destaque é Manataria hercyna hercyna (Hübner), considerada rara na região noroeste do Rio Grande do Sul (BieZAnko 1960a; Giovenardi et al. 2008) e na Argentina (NuÑEz-Bustos 2010). A subespécie voa somente em locais sombrios e úmidos, possui hábitos crepusculares, sendo considerada indicadora de ambientes preservados (BROWN \& FREITAS 2000).

A única espécie considerada dominante nos fragmentos foi Godartiana muscosa (Butler) (10,13\%). As borboletas desta espécie preferem locais sombrios perto de trilhas e pousam em baixa altura ou no solo (CANALS 2003). Segundo MARín et al. (2009) a qualidade da matriz e a persistência das espécies são afetadas pela intensificação da agricultura. $\mathrm{O}$ autor afirma que a agricultura de forma intensiva pode influenciar a composição da fauna e favorecer a alta dominância de somente uma espécie, o que pode explicar a dominância de poucos grupos no estudo. Considerando que as borboletas são afetadas principalmente pelos impactos das atividades agrícolas (BONEBRAKE et al. 2010), o modo como a matriz influencia a fauna pode variar entre diferentes espécies ou assembleias, assim em geral, os distúrbios beneficiam as espécies generalistas e prejudicam as espécies especialistas (LiTTLEWOod et al. 2011). De forma geral, as espécies dominantes podem ser consideradas generalistas, pois podem ser encontradas em diversos tipos de ambientes e fisionomias vegetais, desde os florestados e até aqueles perturbados pela ação antrópica (RuszczyK, 1998; Morais et al. 2007; Silva et al. 2007; SOAREs et al. 2012). Das dez espécies citadas como mais abundantes para o Rio Grande do Sul (Morais et al. 2007), Dryas iulia alcionea (Cramer), Hermeuptychia hermes (Fabricius) e Paryphthimoides phronius (Godart) foram registradas neste estudo, mas nenhuma delas foi considerada dominante. Hypothyris euclea laphria (Doubleday), amostrada em PMAB, foi registrada pela primeira vez no Rio Grande do Sul através da coleta de um único exemplar (Tabela 1). Esta espécie havia sido registrada até o momento na região centro-oeste Paranaense (Dolibaina et al. 2011).

Do total de espécies registradas 23 (42,94\% - seis Biblidinae, três Charaxinae, um Liminitidinae, dois Morphinae, um Nymphalinae e nove Satyrinae) ocorreram nos dois fragmentos, seis $(12,24 \%$ um Ithomiinae, dois Nymphalinae e três Satyrinae) ocorreram somente em SS e 20 (40,82\% - sete Biblidinae, um Charaxinae, três Ithomiinae, dois Morphinae, um Nymphalinae e seis Satyrinae) ocorreram somente em PMAB. O fragmento de PMAB apresentou mais espécies exclusivas que SS, principalmente devido à riqueza de Satyrinae e Biblidinae. Cabe destacar que a fase larval de Biblidinae consome Euphorbiacea, tais como Actinostemon concolor (Spreng.) Müll. Arg., Alchornea glandulosa Poepp. \& Endl., Sapium glandulatum (Vellozo) Pax, Sebastiana brasiliensis Spreng. e rubrivenium Poepp. \& Endl., espécies estas presentes no PMAB (JACOMASSA 2010).

A curva de suficiência mostrou-se ascendente, representando ser impossível inventariar toda a comunidade de borboletas presente no local, sem o aumento do esforço amostral (Figura 3). Segundo Brown \& Freitas (2000) e SANTOS (2003), em ambientes tropicais, raramente a curva se estabiliza. Os estimadores de riqueza Jackknife 1 (54 espécies, indica que foi amostrado 90,74\% da diversidade total), Jackknife 2 (6o espécies, indica que foi amostrado 81,67\% da diversidade total) e Bootstrap (50 espécies, indica que foi amostrado $98 \%$ da diversidade total) corroboram com curva do coletor, indicando a necessidade da continuidade das amostragens. Deste modo, a riqueza observada no presente estudo deve ser tomada como uma subestimativa decorrente do esforço amostral, pois segundo Melo et al. (3003), a riqueza de espécies é fortemente correlacionada com o esforço amostral. Mesmo assim, ressalta-se a riqueza de espécies do presente estudo, pelo mesmo ter sido realizado em dois fragmentos relativamente pequenos de Floresta Estacional Decidual.

Apesar dos locais terem sofrido grande fragmentação nos últimos anos com o crescimento populacional em seu entorno e às atividades pecuárias, as localidades apresentam rica fauna de borboletas. Como não existem estudos com borboletas frugívoras anteriores ao processo de fragmentação, não se torna possível fazer uma comparação dos efeitos da fragmentação. Torna-se necessário, portanto, mais atenção para estudos de paisagens modificadas, situações cada vez mais comuns na atualidade (Moreno \& HalfFter 2001; Ferraz et al. 2009).

A abundância das borboletas no PMAB apresentou-se de forma heterogênea entre os meses de amostragem $\left(\chi^{2}=56,139\right.$; $\mathrm{p}<0,0001)$, com maior abundância no mês de janeiro de 2015 (95 indivíduos), representando 12,5\% dos indivíduos amostrados e menor abundância no mês de junho de 2014 (27 indivíduos), 
Tabela 1. Riqueza, abundância e frequência de espécies de borboletas frugívoras amostradas entre junho de 2014 e março de $2015 .{ }^{*}$ Novo registro de espécie para o Rio Grande do Sul. SS (Santuário de Schoenstatt), PMAB (Parque Municipal Archângelo Busatto).

\begin{tabular}{|c|c|c|c|}
\hline Táxon & SS & PMAB & Frequência \\
\hline \multicolumn{4}{|l|}{ Nymphalidae $(S=49)$} \\
\hline \multicolumn{4}{|l|}{ Biblidinae $(S=14)$} \\
\hline Biblis hyperia nectanabis (Fruhstorfer) & 10 & 36 & 0,061 \\
\hline Callicore hydaspes (Drury) & 2 & 1 & 0,004 \\
\hline Callicore pygas thamyras (Ménetriés) & o & 2 & 0,003 \\
\hline Diaethria c. candrena (Godart) & 4 & 4 & 0,011 \\
\hline Epiphile hubneri Hewitson & 4 & 19 & 0,030 \\
\hline Epiphile o. orea (Hubner) & o & 6 & 0,008 \\
\hline Eunica eburnea Fruhstorfer & o & 3 & 0,004 \\
\hline Eunica tatila bellaria Fruhstorfer & o & 7 & 0,009 \\
\hline Hamadryas a. amphinome (Linnaeus) & o & 2 & 0,003 \\
\hline Hamadryas epinome (C. Felder \& R. Felder) & 11 & 45 & 0,074 \\
\hline Hamadryasf.februa (Hübner) & 1 & 6 & 0,009 \\
\hline Hamadryas f. fornax (Hübner) & o & 3 & 0,004 \\
\hline Myscelia orsis (Drury) & 1 & 8 & 0,012 \\
\hline Temenis laothoe meridionalis Ebert & 0 & 2 & 0,003 \\
\hline Total de indivíduos & 33 & 144 & \\
\hline \multicolumn{4}{|l|}{ Charaxine $(S=4)$} \\
\hline Archaeoprepona chalciope (Hübner) & 5 & 5 & 0,013 \\
\hline Archaeoprepona demophon thalpius (Hübner) & 1 & 7 & 0,011 \\
\hline Memphis moruus stheno (Prittwitz) & 15 & 15 & 0,039 \\
\hline Zaretis itys (Cramer) & o & 1 & 0,001 \\
\hline Total de indivíduos & 21 & $\mathbf{2 8}$ & \\
\hline \multicolumn{4}{|l|}{ Ithomiinae $(S=4)$} \\
\hline Dircenna dero celtina Burmeister & o & 1 & 0,001 \\
\hline Hypothyris euclea laphria (Doubleday)* & o & 1 & 0,001 \\
\hline Pseudoscada erruca (Hewitson) & 1 & o & 0,001 \\
\hline Pteronymia sylvo (Geyer) & o & 2 & 0,003 \\
\hline Total de indivíduos & $\mathbf{1}$ & 4 & \\
\hline \multicolumn{4}{|l|}{ Limenitidinae $(S=1)$} \\
\hline Adelpha syma (Godart) & 2 & 1 & 0,004 \\
\hline Total de indivíduos & 2 & $\mathbf{1}$ & \\
\hline \multicolumn{4}{|l|}{ Morphinae $(S=4)$} \\
\hline Eryphanis reevesii Doubleday & & 1 & 0,001 \\
\hline Morpho a. aega (Hübner) & o & 1 & 0,001 \\
\hline Morpho helenor achillides C. Felder \& R.Felder & 7 & 43 & 0,068 \\
\hline Opoptera aorsa (Godart] & o & 1 & 0,001 \\
\hline Total de indivíduos & 9 & 46 & \\
\hline \multicolumn{4}{|l|}{ Nymphalinae $(S=4)$} \\
\hline Dryas iulia alcionea (Cramer) & o & 1 & 0,001 \\
\hline Hypanartia bella (Fabricius) & 1 & 0 & 0,001 \\
\hline Smyrna b. blomfildia (Fabricius) & 4 & 10 & 0,018 \\
\hline Vanessa braziliensis (Moore & 1 & $\mathrm{O}$ & 0,001 \\
\hline Total de indivíduos & 6 & 11 & \\
\hline \multicolumn{4}{|l|}{ Satyrinae $(S=18)$} \\
\hline Eteona tisiphone (Boisduval) & 3 & 1 & 0,005 \\
\hline Forsterinaria necys (Godart) & o & 30 & 0,039 \\
\hline Forsterinaria quantius (Godart) & 36 & 25 & 0,080 \\
\hline Godartiana muscosa (Butler) & 77 & o & 0,101 \\
\hline Hermeuptychia hermes (Fabricius) & 1 & 13 & 0,018 \\
\hline Manataria h. hercyna (Hübner) & 5 & 8 & 0,018 \\
\hline Pareuptychia summandosa (Gosse) & 1 & 14 & 0,020 \\
\hline Paryphthimoides phronius (Godart) & o & 10 & 0,013 \\
\hline Paryphthimoides poltys (Prittwitz) & 2 & 69 & 0,093 \\
\hline Paryphthimoides sp. & 46 & o & 0,061 \\
\hline Pseudodebis euptychia (Butler) & 0 & 4 & 0,005 \\
\hline Praepedaliodes phanias (Hewitson) & 2 & 8 & 0,013 \\
\hline Splendeuptychia libitina (Butler) & 2 & 2 & 0,005 \\
\hline Taygetis acuta Weymer & o & 31 & 0,041 \\
\hline Taygetis ypthima (Hubner) & 2 & 0 & 0,003 \\
\hline Taygetis sp. & 2 & 5 & 0,009 \\
\hline Yphthimoides celmis (Godart) & o & 26 & 0,034 \\
\hline Yphthimoides straminea (Butler) & $\mathrm{O}$ & 29 & 0,038 \\
\hline Total de indivíduos & 179 & 275 & \\
\hline
\end{tabular}




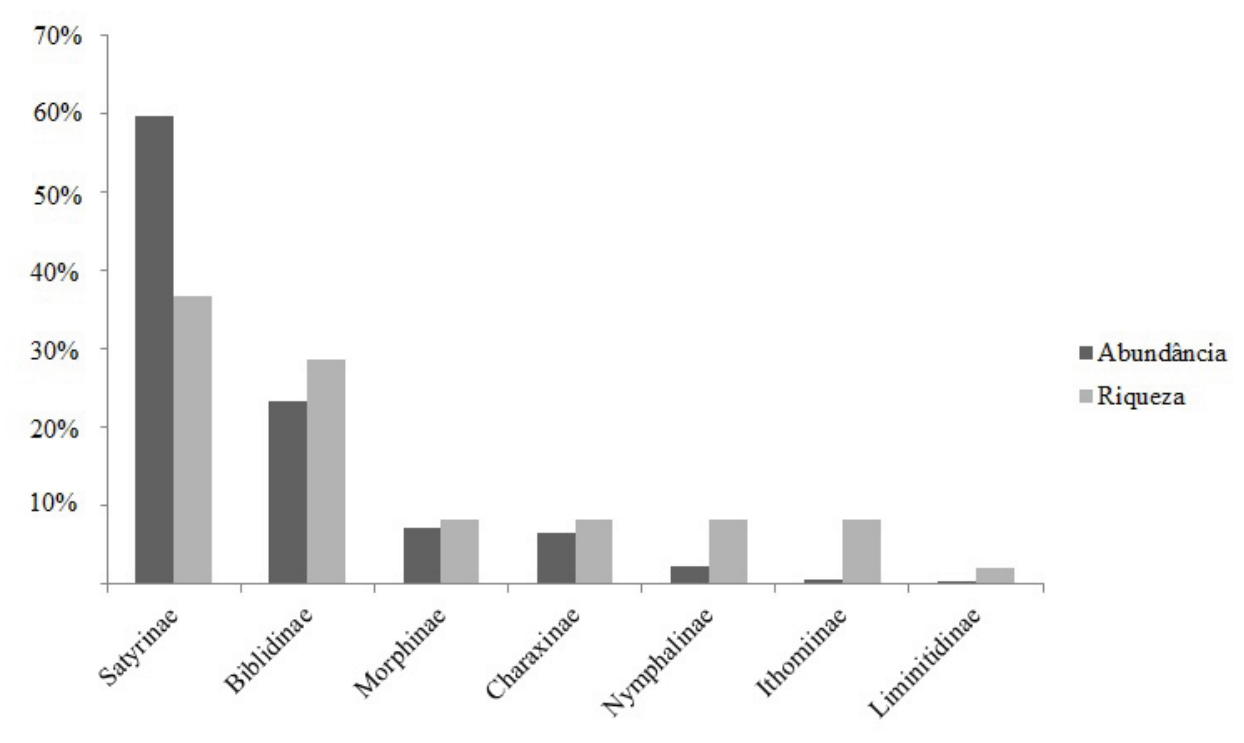

Figura 2. Riqueza e abundância de subfamílias de borboletas frugívoras amostradas entre junho de 2014 e março de 2015 no Parque Municipal Archângelo Busatto e Santuário de Schoesntatt.

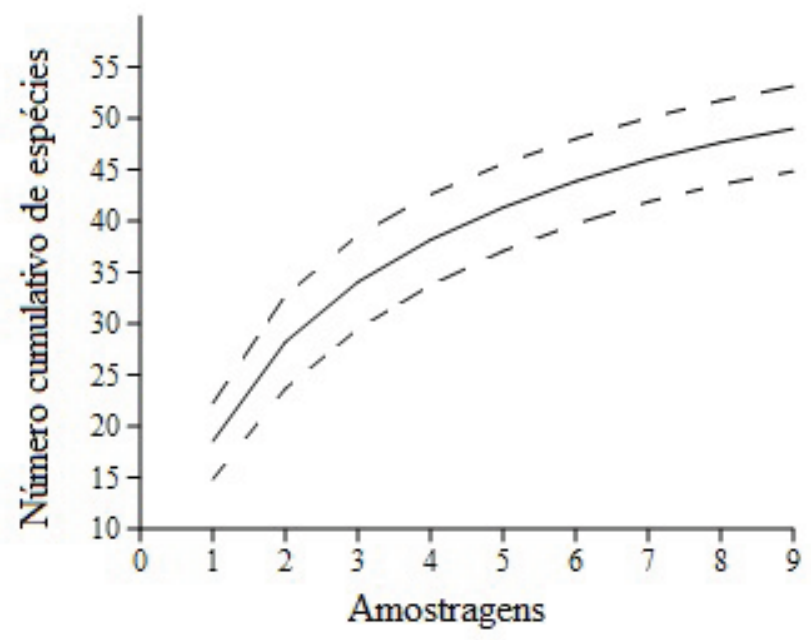

Figura 3. Curva de suficiência amostral para os dois fragmentos, das comunidades de borboletas frugívoras, amostradas entre junho de 2014 e março de 2015 no Parque Municipal Archângelo Busatto e Santuário de Schoesntatt. As linhas pontilhadas indicam o intervalo de confiança (95\%).

representando $3,5 \%$ dos indivíduos amostrados. Da mesma forma, a abundância de borboletas apresentou-se heterogênea no SS $\left(\chi^{2}=442,012 ; p<0,0001\right)$, mas com maior abundância no mês de agosto de 2014 (165 indivíduos), representando 21,71\% dos indivíduos amostrados e menor abundância no mês de janeiro de 2015 (quatro indivíduos), representando 0,53\% dos indivíduos amostrados. A maior abundância em janeiro e menor abundância em junho no PMAB coincidem com os períodos de altas e baixas temperaturas, respectivamente na região. Por sua vez, em SS a maior abundância foi em agosto e menor abundância em janeiro, coincidindo com os períodos de baixas e altas temperaturas, respectivamente. Para a confirmação de quais variáveis inferiram esta diversidade, necessita-se a inclusão de outros fatores climáticos, como luminosidade local e pluviosidade, pois as borboletas são organismos sensíveis ao tempo e alterações microclimáticas locais (EHRLICH \& MURPHY 1987; Brown Jr. \& Freitas 1999; Kitahara 2004).

O presente estudo, apesar de ter se constituído em um inventariamento de curto prazo, descreveu uma fauna de borboletas frugívoras com riqueza de espécies e abundância bastante representativas no noroeste do Rio Grande do Sul. Os dados indicam um possível acréscimo do número de espécies com aumento no esforço amostral e os padrões de composição podem ser utilizados na tomada de decisões de manejo e conservação das áreas (Uehara-Prado et al. 2004). Satyrinae foi a subfamília mais representativa em riqueza e abundância de espécies em ambos os fragmentos e a abundância de espécies mostrou-se diferenciada entre os meses amostrados.

Concluindo-se, os estudos enfocando especificamente as borboletas frugívoras são incipientes no noroeste do Rio Grande do Sul e recomenda-se intensificar estudos semelhantes, para que possam gerar informações necessárias para a conservação deste grupo e os hábitats associados.

\section{AGRADECIMENTOS}

Agradecemos a Universidade Regional Integrada do Alto Uruguai e das Missões- campus Frederico Westphalen, pela bolsa de iniciação científica concedida a esta pesquisa.

\section{REFERÊNCIAS}

Bellaver, J., C.A. Iserhard, J.P. Santos, A.K. Silva, M. Torres, R.R. Siewert, A. Moser, \& H.P. Romanowski, 2012. Butterflies (Lepidoptera: Papilionoidea and Hesperioidea) from Swamp 
forests and Restinga forests at the southern Brazilian Coastal Plain. Biota Neotropica, 12: 181-190.

Biezanko, C.M., 1960a. Satyridae, Morphidae et Brassolidae da Zona Sueste do Rio Grande do Sul. Arquivos de Entomologia, Ser. A: 1-13.

Biezanko, C.M., 196ob. Satyridae, Morphidae et Brassolidae da Zona Missioneira do Rio Grande do Sul. Arquivos de Entomologia, Ser. B: 1-10.

Bonebrake, T.C., L.C. Ponisio, C.L. Boggs \& P.R. Ehrlich, 2010. More than just indicators: a review of tropical butterfly ecology and conservation. Biological Conservation, 143: 18311841. DOI: https://doi.org/10.1016/j.biocon.2010.04.044.

Bonfantti D., R.A. Di-Mare \& R. Giovenardi, 2009. Butterflies (Lepidoptera: Papilionoidea and Hesperioidea) from two forest fragments in northern Rio Grande do Sul, Brazil. Check List, 5: 819-829. DOI: https://doi.org/10.15560/5.4.819.

Brancher, D. \& M. Roza-Gomes, 2012. Survey of edaphic fauna in forest fragment in the municipality of Anchieta (SC, Brazil). Biota Neotropica, 12: 94-98. DOI: https://doi.org/10.1590/s167606032012000300010.

Brown Jr., K.S. \& A.V.L. Freitas, 2000. Atlantic Forest Butterflies: Indicators for Landscape Conservation. Biotropica 32: 934956. DOI: https://doi.org/10.1646/0006-3606(2000)032[0 934:afbifl]2.0.co;2.

Brown, K.S. \& A.V.L. Freitas, 1999. Lepidoptera, p. 225-245, In: Brandão, C.R.F. \& E.M. Cancello (Eds). Biodiversidade do Estado de São Paulo, Brasil: invertebrados terrestres. São Paulo, FAPESP, 279 p.

Canals G., 2000. Mariposas Bonaerenses. Buenos Aires, L.O.L.A. $347 \mathrm{p}$.

Canals, G., 2003. Mariposas de Misiones. Buenos Aires, L.O.L.A. $492 \mathrm{p}$.

Carporale, A., B. Moreno, N.O. Mega \& H.P. Romanowski, 2015 Butterflies (Lepidoptera: Papilionoidea and Hesperioidea) of the Banhado dos Pachecos Wildlife Refuge, Uruguayan Savanna Ecoregion, Rio Grande do Sul state, Brazil. Check List, 11: 1813. DOI: https://doi.org/10.1556o/11.6.1813.

Carvalho, A.P.S., \& A.B.B. Morais, 2015. Borboletas associadas a ambientes de restinga no extremo sul do Brasil (Lepidoptera: Papilionoidea), SHILAP Revista de Lepidopterología, 43: 349-363.

Carvalho, A.P.S., G. Piovesan, A.B.B. Morais, 2015. Butterflies (Lepidoptera: Papilionoidea) of grassland areas in the Pampa biome, southern Brazil. Check List, 11: 1772. DOI: https://doi.org/10.15560/11.5.1772.

Daily, G.C. \& P.R. Ehrlich, 1995. Preservation of biodiversity in small rainforest patches: rapid evaluations using butterfly trapping. Biodiversity and Conservation, 4:35-55. DOI: https://doi.org/10.1007/bfo0115313.

Dessuy, M.B. \& A.B.B. Morais, 2007. Diversidade de borboletas (Lepidoptera: Papilionoidea e Hesperioidea) em fragmentos de Floresta Estacional Decidual em Santa Maria, Rio Grande do Sul, Brasil. Revista Brasileira de Zoologia, 24: 108-120. DOI: https://doi.org/10.1590/s0101-81752007000100014.

DeVries P.J., 1997. The Butterflies of Costa Rica and their Natural History. II: Riodinidae. Princeton, New Jersey: Princeton University Press. 288 p.

DeVries, P.J. \& T.R. Walla, 2001. Species diversity and community structure in neotropical fruit-feeding butterflies. Biological Journal of the Linnean Society, 74: 1-15. DOI: https://doi.org/10.1006/bijl.2001.0571.

DeVries, P.J., T.R. Walla, \& H.F. Grenney, 1999. Species diversity in spatial and temporal dimensions of fruit-feeding butterflies from two Ecuadorian rainforests. Biological Journal of the Linnean Society, 68:333-353. DOI: https://doi.org/10.1111/ j.1095-8312.1999.tbo1175.x.

Dolibaina, D.R., O.H.H. Mielke \& M.M. Casagrande, 2011. Borboletas (Papilionoidea e Hesperioidea) de Guarapuava e arredores, Paraná, Brasil: um inventário com base em
63 anos de registros. Biota Neotropica, 11: 341-354. DOI: https://doi.org/10.1590/s1676-06032011000100031.

Ehrlich, P.R. \& D.D. Murphy, 1987. Conservation lessons from longterm studies of checkerspot butterflies. Conservation Biology, Boston, 1: 122-131. DOI: https://doi.org/10.1111/ j.1523-1739.1987.tbooo21.x.

Ferraz, A.C.P., B.A. Gadelha \& V.M. Aguiar-Coelho, 2009. Análise faunística de Calliphoridae (Diptera) da Reserva Biológica do Tinguá, Nova Iguaçu, Rio de Janeiro. Revista Brasileira de Entomologia, 53: 620-628. DOI: https://doi.org/10.1590/ s0085-56262009000400012.

Freitas, A.V. L., R.B. Francini \& K.S. Brown, 2003. Insetos como indicadores ambientais. In L. Cullen Junior, C. ValladaresPádua \& R. Rudran. Métodos de estudos em biologia da conservação e manejo da vida silvestre: Editora da UFPR, Curitiba, $667 \mathrm{p}$.

Freitas, A.V.L., O.H.H. Mielke, A. Moser, K.L. SilvaBrandão \& C.A. Iserhard, 2011. A New Genus and Species of Euptychiina (Lepidoptera: Nymphalidae: Satyrinae) from Southern Brazil. Neotropical Entomology 40: 231-237. DOI: https://doi.org/10.1590/s1519$566 \times 2011000200012$.

Giovenardi, R., R.A. Di Mare, J. Sponchiado, S.H. Roani, F.A.F. Jacomassa, A.B. Jung \& M.A. Porn. 2008. Diversidade de Lepidoptera (Papilionoidea e Hesperioidea) em dois fragmentos de floresta no município de Frederico Westphalen, Rio Grande do Sul, Brasil. Revista Brasileira de Entomologia, 52: 599-605. DOI: https://doi.org/10.1590/ s0085-56262008000400010.

Hammer, Ø., D.A.T. Harper \& P.D. Ryan, 2001. PAST: Paleontological Statistics software package for education data analysis. Paleontologia Electronica, 4: 9.

Iserhard, C.A. \& H.P. Romanowski, 2004. Lista de espécies de borboletas (Lepidoptera: Papilionoidea e Hesperioidea) da região do vale do rio Maquiné, Rio Grande do Sul, Brasil. Revista Brasileira de Zoologia, 21: 649-662. DOI: https://doi.org/10.1590/s0101-81752004000300027.

Iserhard, C.A., M.T. Quadros, H.P. Romanowski \& M.S. Mendonça Jr., 2010. Borboletas (Lepidoptera: Papilionoidea e Hesperioidea) ocorrentes em diferentes ambientes na Floresta Ombrófila Mista e nos Campos de Cima da Serra do Rio Grande do Sul, Brasil. Biota Neotropica, 10: 309-320. DOI: https://doi.org/10.1590/s1676-06032010000100026.

Jacomassa, F.A.F., 2010. Espécies arbóreas nativas da mata ciliar da bacia hidrográfica do rio lajeado tunas, na região do alto Uruguai, RS. Biodiversidade Pampeana, 8: 1-6.

Kitahara, M., 2004. Butterfly community composition and conservation in and around a primary woodland of Mont Fuji, central Japan. Biodiversity and Conservation, London, 13: 917-942. DOI: https://doi.org/10.1023/ b:bioc.0000014462.83594.58.

Lamas, G., 2004. Atlas of Neotropical Lepidoptera. Association for Tropical Lepidoptera.Gainesville: Scientific Publishers, USA, $439 \mathrm{p}$.

Lemes, R., A.P.S. Carvalho, T.C. Ribeiro \& A.B.B. Morais, 2015. Borboletas de áreas verdes urbanas de Santa Maria, sul do Brasil (Lepidoptera: Papilionoidea). SHILAP Revista de Lepidopterología, 43: 95-111.

Lemes, R., C.D. Ritter \& A.B.B. Morais, 2008. Borboletas (Lepidoptera: Hesperioidea e Papilionoidea) visitantes florais no Jardim Botânico da Universidade Federal de Santa Maria, Santa Maria, RS, Brasil. Biotemas, 21: 91-98. DOI: https://doi.org/10.5007/2175-7925.2008v21n4p91.

Link, D. \& A. Alvarez-Filho, 1979. Palmeiras atacadas por lagartas de Brassolidae (Lepidoptera) em Santa Maria, RS. Ciência Rural, 9: 221-225.

Link, D., C.M. Biezanko, M.F. Tarragó \& S. Carvalho, 1980. Lepidoptera de Santa Maria e arredores. II: Morphidae e Brassolidae. Ciência Rural, 10: 191-195.

Littlewood, N.A., A.J.A. Stewart, \& B.A.Woodcock, 2011. Science into practice - how can fundamental science contribute 
to better management of grasslands for invertebrates? Insect Conservation and Diversity, 5: 1-8. DOI: https://doi.org/10.1111/j.1752-4598.2011.00174.x.

Maluf, J.R.T., 2000. Nova classificação climática do Estado do Rio Grande do Sul. Revista Brasileira de Agrometeorologia, 8: $141-150$.

Marchiori, M.O. \& H.P. Romanowski, 2006. Borboletas (Lepidoptera, Papilionoidea e Hesperioidea) do Parque Estadual do Espinilho e entorno, Rio Grande do Sul, Brasil. Revista Brasileira de Zoologia, 23: 443-454. DOI: https://doi.org/10.1590/s0101-81752006000400007.

Marchiori, M.O., H.P. Romanowski \& M. Souza-Mendonça Jr., 2014. Mariposas en dos ambientes forestales contrastantes en el sur de Brasil (Lepidoptera: Hesperioidea \& Papilionoidea). SHILAP Revista de Lepidopterología, 42: 221-236.

Marín, L., J.L. León-Cortes \& C. Stefanescu, 2009. The effect of an agro-pasture landscape on diversity and migration patterns of frugivorous butterflies in Chiapas, Mexico. Biodiversity Conservation, 18: 919-934. DOI: https://doi.org/10.1007/ s10531-008-9540-z.

Melo, A.S., R.A.S.Pereira, A.J. Santos, G.J. Shepherd, G. Machado, H.F. Medeiros \& R.J. Sawaya, 2003. Comparing species richness among assemblages using sample units: Why not use extrapolation methods to standardize different sample sizes? Oikos, 101: 398-410. DOI: https://doi.org/10.1034/j.16000706.2003.11893.x.

Ministério do Meio Ambiente, 2010. Mata Atlântica: manual de adequação ambiental. (Série Biodiversidade, 35), 96 p.

MinistériodoMeioAmbiente,2017. MataAtlântica.Disponívelem: <http://www.mma.gov.br/biomas/mata-atl\%C3\%A2ntica emdesenvolvimento $>$. [Acessado em: 04.iv.2018].

Morais, A.B.B., H.P. Romanowski, C.A. Iserhard, M.O. Marchiori \& R. Segui, 2007. Mariposas del sur de Sudamérica (Lepidoptera: Hesperioidea y Papilionoidea). Ciência \& Ambiente, 35: 29-46.

Morais, A.B.B., R. Lemes \& C.D. Ritter, 2012. Borboletas (Lepidoptera: Hesperioidea e Papilionoidea) de Val de Serra, região central do Rio Grande do Sul, Brasil. Biota Neotropica, 12: 175-183. DOI: https://doi.org/10.1590/ s1676-06032012000200017.

Moreno, C.E. \& G. Halffter, 2001. Spatial and temporal analysis of $\mathrm{a}, \mathrm{b}$ and $\mathrm{g}$ diversities of bats in a fragmented landscape. Biodiversity and Conservation, 10: 367-382.

Núñez-Bustos, E.O., 2010. Mariposas de la ciudad de Buenos Aires y alrededores. Buenos Aires, Vásquez MazziniEditores $264 \mathrm{p}$.

Paz, A.L.G., H.P. Romanowski \& A.B.B. Morais, 2008. Nymphalidae, Papilionidae e Pieridae (Lepidoptera: Papilionoidea) da Serra do Sudeste do Rio Grande do Sul, Brasil. Biota Neotropica, 8: 22-29. DOI: https://doi.org/10.1590/s1676-06032008000100017.

Paz, A.L.G., H.P. Romanowski, \& A.B.B. Morais, 2014. Borboletas frugívoras do centro-oeste do Rio Grande do Sul (Lepidoptera: Nymphalidae). SHILAP Revista de Lepidopterología, 41: 409-422.

Pedrotti, V.S., M.P. Barros, H.P. Romanowski \& C.A. Iserhard, 2011. Borboletas frugívoras (Lepidoptera: Nymphalidae) ocorrentes em um fragmento de Floresta Ombrófila Mista no Rio Grande do Sul, Brasil. Biota Neotropica, 11: 385-19o. DOI: https://doi.org/10.1590/s1676-06032011000100036.

Penz, C., I. Garzón \& N. Mohammadin, 2007. PenzLab. University of New Orleans. Disponível em: <http://fs.uno.edu/cpenz/>. [Acesso em: 08.viii.2009].

Pinheiro, C.E.G. \& J.V.C. Ortiz, 1992. Communities of fruitfeeding butterflies along a vegetation gradient in central Brazil. Journal of Biogeography, 19: 505-511. DOI: https://doi.org/10.2307/2845769.

Piovesan, G. \& A.B.B. Morais, 2015. Borboletas de fragmentos de Mata Atlântica da região central do Rio Grande do Sul, Brasil (Lepidoptera: Papilionoidea). SHILAP Revista de Lepidopterología, 43: 199-216.
Quadros, F.C., A.L. Dorneles \& E. Corseuil, 2004. Ninfalídeos (Lepidoptera, Nymphalidae) ocorrentes no norte da Planície Costeira do Rio Grande do Sul. Biociências, 12: 147-164.

Ribeiro, M.C., J.P. Metzger, A.C. Martensen, F.J. Ponzoni \& M.M. Hirota, 2009. The Brazilian Atlantic Forest: How much is left, and how is the remaining forest distributed? Implications for conservation. Biological Conservation, 142: 1141-1153. DOI: https://doi.org/10.1016/j.biocon.2009.02.021.

Ritter, C.D., R. Lemes, A.B.B. Morais \& C.S. Dambros, 2011. Borboletas Lepidoptera: Hesperioidea e Papilionoidea) de fragmentos de Floresta Ombrófila Mista, Rio Grande do Sul, Brasil. Biota Neotropica, 11: 361-368. DOI: https://doi.org/10.1590/s1676-06032011000100033.

Romanowski, H.P., C.A. Iserhard \& S.M. Hartz, 2009. Borboletas da Floresta com Araucária, p. 229-240. In: Fonseca, C.R., A.F. Souza, A.M. Leal-Zanchet, T. Dutra, A. Backes \& G. Ganade. Floresta com Araucária: ecologia, conservação e desenvolvimento sustentável: Ribeirão Preto, Holos 326 p.

Rosa, P.L.P., E.Q. Chiva \& C.A. Iserhard, 2011. Borboletas (Lepidoptera: Papilionoidea e Hesperioidea) do sudoeste do pampa brasileiro, Uruguaiana, Rio Grande do Sul, Brasil. Biota Neotropica, 11: 355-360. DOI: https://doi.org/10.1590/ s1676-06032011000100032.

Ruszczyk, A., 1998. Borboletas: indicadoras da qualidade ambiental, p. 69-70. In: R. Menegat (Coord.). Atlas ambiental de Porto Alegre: Porto Alegre, Editora da Universidade/ UFRGS. 228 p.

Sackis, G.D. \& A.B.B. Morais, 2008. Borboletas (Lepidoptera: Hesperioidea e Papilionoidea) do campus da Universidade Federal de Santa Maria, Santa Maria, Rio Grande do Sul. Biota Neotropica, 8: 21-28. DOI: https://doi.org/10.1590/ s1676-06032008000100018.

Santos, J.A., 2003. Estimativa de riqueza em espécies, p. 19-41. In: Cullen Jr., L., R. Rudran \& C. Valladares-Pádua (Eds.). Métodos de estudo em biologia da conservação e manejo da vida silvestre. Curitiba: Editora da UFPR, 1942 p.

Santos, J.P., C. A Iserhard, M.O. Teixeira \& H.P. Romanowski, 2011. Fruit-feeding butterflies guide of subtropical Atlantic Forest and Araucaria Moist Forest in State of Rio Grande do Sul, Brazil. Biota Neotropica, 11: 253-274. DOI: https://doi.org/10.1590/s1676-06032011000300022.

Silva, A.R.M., G.G Landa \& R.F. Vitalino, 2007. Borboletas (Lepidoptera) de um fragmento de mata urbano em Minas Gerais, Brasil. Lundiana, 8: 137-142.

Silva, J.M.S., S.K. Cunha, E.J.E. Silva \& F.R.M. Garcia, 2013. Borboletas frugívoras (Lepidoptera: Nymphalidae) no Horto Botânico Irmão Teodoro Luis, Capão do Leão, Rio Grande do Sul, Brasil. Biotemas, 26: 87-95. DOI: https://doi.org/10.5007/2175-7925.2013v26n1p87.

Soares, G.R., A.A.P. Oliveira \& A.R.M. Silva, 2012. Borboletas (Lepidoptera: Papilionoidea e Hesperioidea) de um parque urbano em Belo Horizonte, Minas Gerais, Brasil. Biota Neotropica, 12: 209-217. DOI: https://doi.org/10.1590/ s1676-06032012000400022.

Spaniol, R.L. \& A.B.B. Morais, 2015. Borboletas frugívoras em uma área de transição ecológica do sul do Brasil. SHILAP Revista de Lepidopterología, 43: 27-40.

Teston, J.A. \& E. Corseuil, 2002a. Borboletas (Lepidoptera, Rhopalocera) ocorrentes no CentrodePesquisas eConservação da Natureza Pró-Mata. 3. Nymphalidae. Divulgações do Museu de Ciências e Tecnologia, UBEA/PUCRS, 7: 1-208.

Teston, J.A. \& E. Corseuil, 2002b. Ninfalídeos (Lepidoptera, Nymphalidae) ocorrentes no Rio Grande do Sul, Brasil. Biociências, 10: 75-84.

Teston, J.A., \& E. Corseuil, 2008a. Ninfalídeos (Lepidoptera, Nymphalidae) ocorrentes no Rio Grande no Sul, Brasil. Parte IV. Apaturinae e Charaxinae. Biociências, 16: 28-32.

Teston, J.A., \& E. Corseuil, 2008b. Ninfalídeos (Lepidoptera, Nymphalidae) ocorrentes no Rio Grande do Sul, Brasil. Parte V. Biblidinae e Limenitidinae. Biociências, 16: 33-41. 
Teston, J.A., \& E. Corseuil, 2008c. Ninfalídeos (Lepidoptera, Nymphalidae) ocorrentes no Rio Grande do Sul, Brasil. Parte VI. Nymphalinae e Satyrinae. Biociências, 16(1): 42-51.

Thiele, S.C., O. Milcharek, F.L. Santos, L.A. Kaminski, 2014. Butterflies (Lepidoptera: Hesperioidea and Papilionoidea) of Porto Mauá, Upper Paraná Atlantic Forest Ecoregion, Rio Grande do Sul State, Brazil. Biota Neotropica, 14: e20130006. DOI: https://doi.org/10.1590/1676-06032014000613.

Uehara-Prado, M., A.V.L. Freitas, R.B. Francini \& K.S. Brown, 2004. Guia das borboletas frugívoras da Reserva Estadual do Morro Grande e região de Caucaia do Alto, Cotia (São Paulo). Biota Neotropica, 4: 1-25. DOI: https://doi.org/10.1590/ s1676-06032004000100007.
Uehara-Prado, M., K.S. Brown \& A.V.L. Freitas, 2005. Biological traits of frugivorous butterflies in a fragmented and a continuous landscape in the south Brazilian Atlantic Forest. Journal of Lepidopterists Society, 59: 96-106.

Wahlberg, N., J. Leneveu, U. Kodandaramaiah, C. Pena, S. Nylin, A.V.L. Freitas \& A.V.Z. Brower, 2009. Nymphalid butterflies diversity following neardemise at the Cretaceous/Tertiary boundary. Proceedings of the Royal Society B: Biological Sciences, 276: 4295-4302. DOI: https://doi.org/10.1098/ rspb.2009.1303.

\section{Suggestion citation:}

Pinho da Silva, T., W.F. Pias \& R. Giovenardi, 2018. Borboletas frugívoras de fragmentos de Mata Atlântica no noroeste do Rio Grande do Sul, Brasil (Lepidoptera: Nymphalidae). EntomoBrasilis, 11 (2): 95-102.

Available on: doi:10.12741/ebrasilis.v11i2.773
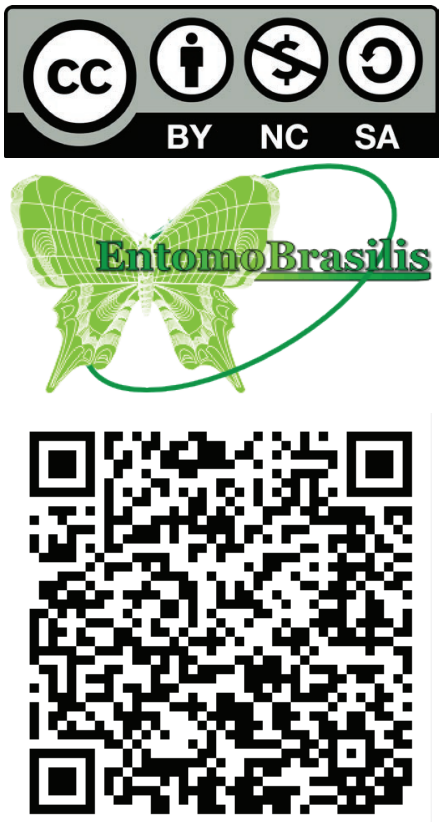